PROCEEDINGS OF THE AMERICAN MATHEMATICAL SOCIETY

Volume 126, Number 3, March 1998, Pages 823-825

S $0002-9939(98) 04107-0$

\title{
THE ORLICZ-PETTIS THEOREM FOR TOPOLOGICAL RIESZ SPACES
}

\author{
LECH DREWNOWSKI AND IWO LABUDA
}

(Communicated by Dale Alspach)

\begin{abstract}
A finitely additive vector measure from a $\sigma$-ring to a Riesz space is countably additive (exhaustive) for all Hausdorff Lebesgue topologies on the range space, or for none of them. In particular, subseries convergent series are the same for all Hausdorff Lebesgue topologies on a Riesz space.
\end{abstract}

Our terminology concerning locally solid topological Riesz spaces is that of [1]. In particular, such a space as well as its topology is said to be Lebesgue if every decreasing net with infimum 0 is topologically convergent to 0 .

A finitely additive measure $\mathbf{m}: \mathcal{R} \rightarrow X$, where $\mathcal{R}$ is a ring of sets and $X$ is a topological vector space, is called exhaustive (strongly bounded) if $\mathbf{m}\left(A_{n}\right) \rightarrow 0$ for each disjoint sequence $\left(A_{n}\right)$ in $\mathcal{R}$.

Let $\mathcal{P}$ denote the power set of $\mathbb{N}$. We recall that $\mathcal{P}$ becomes a complete metric space when equipped with the metric $d(M, N)=\nu(M \Delta N)$ induced by the measure $\nu(N)=\sum_{n \in N} 2^{-n}$. Proposition 1 below is standard and goes back to Saks (see e.g. [4], Proof of Thm. III.7.2 and notes on p. 234); for Proposition 2, see [3, Lemma].

Proposition 1. If $\mathbf{m}: \mathcal{P} \rightarrow X$ is a finitely additive measure, then $\mathbf{m}$ is countably additive iff $\mathbf{m}$ is continuous iff $\mathbf{m}$ has a point of continuity.

Proposition 2. Let $X$ be a metrizable topological vector space. Then a finitely additive measure $\mathbf{m}: \mathcal{P} \rightarrow X$ is exhaustive iff every disjoint sequence $\left(N_{k}\right)$ in $\mathcal{P}$ has a subsequence $\left(M_{k}\right)$ such that $\mathbf{m}$ is countably additive on the $\sigma$-algebra in $\mathbb{N}$ generated by $\left(M_{k}\right)$.

Theorem. Let $\tau_{1}$ and $\tau_{2}$ be two Hausdorff Lebesgue topologies on a Riesz space X, $\mathcal{R}$ a $\sigma$-ring of sets, and $\mathbf{m}: \mathcal{R} \rightarrow X$ a finitely additive measure. If $\mathbf{m}$ is $\tau_{1}$-countably additive (resp. exhaustive), then it is $\tau_{2}$-countably additive (resp. exhaustive).

Proof. Clearly, we may assume $\mathcal{R}=\mathcal{P}$. We proceed by a series of reductions.

(1) In view of [1, Thm. 11.10], both $\tau_{1}$ and $\tau_{2}$ admit Hausdorff Lebesgue extensions to the Dedekind completion of $X$. Thus, we may assume that $X$ is Dedekind complete.

Received by the editors April 16, 1996 and, in revised form, September 9, 1996.

1991 Mathematics Subject Classification. Primary 40A99, 46A40.

Key words and phrases. Subseries convergence, countably additive vector measure, exhaustive vector measure, topological Riesz space, Lebesgue topology.

The paper was written while the first author held a visiting position in the Department of Mathematics, University of Mississippi, in the Spring Semester of 1996. He was also partially supported by the State Committee for Scientific Research (Poland), grant no 2 P301 00307.

(c)1998 American Mathematical Society 
(2) In order to prove that $\mathbf{m}$ is $\tau_{2}$-countably additive (resp. exhaustive), it suffices to show it has this property with respect to each $\tau_{2}$-continuous monotone $F$-seminorm on $X$. Let $p$ be any of such $F$-seminorms, and let $X_{p}$ be its carrier, that is, the band obtained as the disjoint complement to the null-band $p^{-1}(0)$ of $p$. We equip $X_{p}$ with the metric Lebesgue topology determined by $p$ and have to show that the composition of $\mathbf{m}$ with the band projection onto $X_{p}$ is countably additive (resp. exhaustive). Thus, we can assume in what follows that $\tau_{2}$ is metrizable and, consequently, $X$ has the countable sup property [1, 17.8, Remark]. That is, whenever $A \subset X$ and $x=\sup A$ exists, there is a countable subset $A^{\prime}$ of $A$ with $\sup A^{\prime}=x$.

(3) Let $B$ be the band generated by the sequence $u_{n}=\mathbf{m}(\{n\})$. Note that if $\mathbf{m}$ is $\tau_{1}$-countably additive, then $B$ contains the range of $\mathbf{m}$. On the other hand, in the exhaustive case, the assertion will follow easily once it is shown that $u_{n} \rightarrow 0\left(\tau_{2}\right)$. Thus, replacing $\mathbf{m}$ by its composition with the band projection onto $B$, we can assume that $X=B$. Then, as $X$ has also the countable sup property, any disjoint system in $X$ is at most countable.

(4) Now, by [1, Thm. 12.4], $X$ admits a metrizable Lebesgue topology weaker than $\tau_{1}$. Therefore, we may assume that also $\tau_{1}$ is metrizable. So, by Proposition 2, only the countably additive case has to be considered in what follows.

(5) Thus, it remains to prove that $\mathbf{m}$ is $\tau_{2}$-countably additive, or $\tau_{2}$-continuous, when both $\tau_{1}$ and $\tau_{2}$ are metric, and $X$ is generated by the sequence $\left(u_{n}\right)$. Denote $v_{k}=k \sum_{n=1}^{k}\left|u_{n}\right|$, and observe that $x=\sup _{k}\left(x \wedge v_{k}\right)$ for every $x \in X_{+}$. Define maps $\mathbf{m}_{k}: \mathcal{P} \rightarrow X$ by $\mathbf{m}_{k}(N)=\left(\mathbf{m}(N) \wedge v_{k}\right) \vee\left(-v_{k}\right), k=1,2, \ldots$ Each $\mathbf{m}_{k}$ is $\tau_{2}$-continuous because $\tau_{1}$ and $\tau_{2}$ coincide on order intervals [1, Thm. 12.9]. Moreover, as $\tau_{2}$ is Lebesgue, $\mathbf{m}(N)=\tau_{2}-\lim _{k} \mathbf{m}_{k}(N)$ for every $N \in \mathcal{P}$. Therefore, the map $\mathbf{m}: \mathcal{P} \rightarrow\left(X, \tau_{2}\right)$ is of the first Baire class and, consequently, has a point of continuity (see [6], Remark 5 on p. 397). Apply Proposition 1.

Remark. The theorem remains valid if $\tau_{1}$ is any locally solid topology on $X$ such that the topology $\inf \left\{\tau_{1}, \tau_{2}\right\}$ is Hausdorff. It is so, for instance, if $\tau_{1}$ is Fatou (by [1, Thm. 12.7]) or merely $\sigma$-Fatou (by [7, Thm. 2.7 and Cor. 3.12]), or metrizable (by [2, Thms. 3.2 and 3.5]).

For a survey of Orlicz-Pettis type theorems, see [5].

\section{ADDED IN PROOF}

It is important to point out that measures $\mathbf{m}$ as in the Theorem need not be order bounded. We are grateful to Z Z. Lipecki for the following simple example of a countably additive measure $\mathbf{m}: \mathcal{P} \rightarrow L_{2}[0,1]$ which is not order bounded: $\mathbf{m}(N)=\sum_{n=1}^{\infty} n^{-1} 1_{N}(n) r_{n}$, where $\left(r_{n}\right)$ is the Rademacher sequence.

\section{REFERENCES}

1. C. Aliprantis and O. Burkinshaw, Locally solid Riesz spaces, Academic Press, 1978. MR 58:12271

2. MR 81i: 46007

3. L. Drewnowski, Equivalence of Brooks-Jewett, Vitali-Hahn-Saks and Nikodym theorems, Bull. Acad. Polon. Sci., Sér. Sci. Math. Astronom. Phys. 20 (1972), 725-731. MR 47:431

4. N. Dunford and J.T. Schwartz, Linear operators. Part I, Interscience, New York, 1958. MR 22:8302 
5. N.J. Kalton, The Orlicz-Pettis theorem, Contemporary Mathematics 2 (1980), 91-100. MR 82g: 46027

6. K. Kuratowski, Topology, vol. 1, Academic Press, New York and London, 1966. MR 36:840

7. I. Labuda, Submeasures and locally solid topologies on Riesz spaces, Math. Z. 195 (1987), 179-196. MR 88j:46009

Faculty of Mathematics and Computer Science, A. Mickiewicz University, Matejki 48/49, 60-769 Poznań, Poland

E-mail address: drewlech@math.amu.edu.pl

Department of Mathematics, University of Mississippi, University, Mississippi 38677

E-mail address: mmlabuda@vm.cc.olemiss.edu 